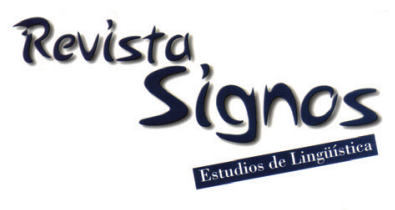

\title{
Aplicabilidad de la tipología de funciones retóricas de las citas al género de la memoria de máster en un contexto transcultural de enseñanza universitaria
}

Applicability of the typology of rhetorical functions of citations to the master's thesis genre in cross cultural context of university teaching

\section{David Sánchez}

UNIVERSITY OF WASHINGTON

EsTADOS UNIDOS

djsan@uw.edu

Recibido: 5-XI-2011 / Aceptado: 3-IX-2012

\section{Resumen}

El objetivo de este artículo es comparar las funciones retóricas que adquieren las citas en 14 memorias de máster escritas por 7 escritores españoles y 7 filipinos en español. Se utiliza una tipología de 9 categorías con el fin de identificar las diferencias retóricas culturales que existen en la práctica de la citación a partir del contraste de este elemento en la cultura filipina y en la española. La metodología utilizada es la del análisis textual del contexto lingüístico en el que aparecen las citas y su clasificación posterior en las 9 categorías señaladas. Los resultados muestran que existen diferencias cuantitativas y cualitativas entre las convenciones culturales de las citas entre ambas nacionalidades. Hay una tendencia mayor por parte del grupo español a utilizar un estilo más argumentativo en la composición de la memoria con respecto al estilo más expositivo de los escritores filipinos, lo que se evidencia en la selección de las funciones retóricas de las citas. Estas diferencias apuntan a la necesidad de desarrollar una didáctica para la enseñanza de las funciones retóricas de las citas que garantice un acercamiento transcultural en esta práctica a los escritores que elaboran un trabajo universitario de investigación en una lengua extranjera.

Palabras Clave: Funciones citas, retórica contrastiva, escritura académica. 


\begin{abstract}
The aim of this paper is to compare the rhetorical functions gathered from the citations of (14) fourteen master's theses written by seven Spanish and seven Philippine authors. A typology of nine categories was used in order to identify the cultural rhetorical differences that exist in the use of citation from the contrast between contrasting this element in the Philippine and Spanish cultures. The methodology used is textual analysis of the linguistic context of these citations and its subsequent classification within these nine categories. The results show that there are quantitative and qualitative differences between the cultural conventions of citations between the two nationalities. The Spanish group shows a higher inclination to a more argumentative style, while the Philippine group, exhibits the use of an expository style of thesis writing. It has been evidenced in the selection of the rhetorical functions of citations. This, therefore, suggests the need to develop didactics to teach rhetorical functions of citations that ensure a cross-cultural approach to postgraduate students who undergo research in a foreign language.
\end{abstract}

Key Words: Citation functions, contrastive rhetoric, academic writing.

\title{
INTRODUCCIÓN
}

Los procesos de citación se conforman en un marco textual que caracteriza al discurso académico. En este sentido, la referencia explícita de antecedentes teóricos es un indicador de suma importancia sobre la relación que existe entre el texto académico y el contexto de conocimiento en el cual se enmarca, por la construcción colaborativa de nuevos saberes entre los escritores y los lectores (Hyland, 2000; Venegas, 2006; Parodi, 2007). Así, se puede definir la citación en el plano textual como la constatación física de la construcción de conocimiento de forma colaborativa a través de intertextos (Bazerman, 2003). Sobre esto, Parodi (2007) opina que la inclusión de elementos de la red de referencias que se establece entre los autores se hará en función de las prácticas estipuladas por cada disciplina, lo que servirá para reforzar las afirmaciones del investigador con las que previamente han emitido otros autores en ese campo de estudio.

Las investigaciones sobre la identificación y la relevancia de las funciones de las citas en el texto académico han experimentado un creciente desarrollo dentro de la Lingüística desde la última década del pasado siglo, especialmente desde que Dong (1996) fijara su mirada en la escritura universitaria de posgrado. Igualmente valiosa ha sido la temprana contribución de Hyland (1999) en este asunto dentro de los estudios de análisis del discurso, en un trabajo que analizaba 80 textos académicos, en el que se comparaban las prácticas de citación de escritores profesionales de ocho disciplinas diferentes. En estos años Cumming (1998) y Dudley-Evans (2000), en sendas revisiones sobre las perspectivas futuras de la escritura, vaticinaban los nuevos caminos de la investigación dentro del análisis de textos, entre los que concedían 
una atención prioritaria al estudio de las citas. Las investigaciones posteriores en este campo (Thompson, 2001, 2005; Petrić, 2007; Samraj, 2008; Harwood, 2009) desde una perspectiva lingüística vienen a darles la razón. Sin embargo, no hemos de obviar que antes de que el análisis textual y el análisis del discurso comenzaran a interesarse por esta materia, ya mucho tiempo antes otras disciplinas las habían convertido en su objeto principal de estudio, como la Sociología de la Ciencia o las Ciencias de la Información.

Dentro de la vía didáctica iniciada por Dong (1996) se incluye el presente trabajo de investigación, que compara las funciones retóricas de las citas que utilizan un grupo de escritores filipinos y otro de españoles en el género académico de la memoria de máster escrita en español como L2 y como L1 respectivamente. En este estudio se han analizado los patrones discursivos de la memoria en función de los tipos de cita empleados en cada una de las partes, desde los presupuestos de la Retórica Contrastiva. Dentro del ámbito de esta disciplina, se han llevado a cabo numerosos estudios sobre los géneros (Connor, 1996), pero apenas existen algunos trabajos en el contraste cultural de las citas (Mayor Serrano, 2006; Okamura, 2008), y ninguno contrastivo sobre estudiantes no nativos aprendiendo una LE. Solo Dong (1996), Petrić (2007), Schembri (2009), Wette (2010) y Mansourizadeh y Ahmad (2011) han abordado la dificultad que encuentran los estudiantes universitarios en la práctica de la citación al componer en una L2.

Con el fin de conocer mejor las repercusiones que tiene en la enseñanza universitaria el uso de las funciones retóricas de las citas en un contexto de lengua extranjera, se han comparado en este artículo 7 memorias de máster escritas por estudiantes nativos con otras 7 elaboradas por estudiantes filipinos en lengua española en los usos que ambos grupos hacen de las citas. La existencia de maneras diferentes de usar las funciones de las citas entre los dos grupos analizados supondría una variación cultural en el uso de las funciones retóricas de las citas. La verificación de esta hipótesis en nuestro estudio contribuiría a la defensa que vienen haciendo distintos autores (Dong, 1996; Thompson, 2005; Petrić, 2007; Schembri, 2009; Wette, 2010) sobre la necesidad de aplicar-mediante la instrucción formal- una didáctica concreta con el fin de corregir las desviaciones impuestas por las convenciones existentes en la lengua nativa a la hora de escribir en una lengua extranjera.

\section{Marco teórico}

El análisis de citas ha sido un campo de estudio profusamente investigado por los sociólogos de la información y los científicos de la información desde los años 50 (Merton, 1942; Garfield, 1955), si bien dicho análisis respondía a un propósito bien distinto al que se marcarían los lingüistas en las décadas siguientes. Dentro de las Ciencias de la Información, la atención sobre las citas se centraba en la cuantificación de las mismas, con el fin de evaluar los trabajos científicos publicados, mientras que en la Sociología de la Ciencia se establecía un debate sobre las funciones de las citas (Gilbert, 1977; Garfield, 1979, 1998; Cronin, 1998), al que se sumarían también con 
posterioridad las voces de algunos lingüistas (Hyland, 1999; Laca, 2001). En este sentido, se distinguían dos posturas enfrentadas', la 'normativa' (Garfield, 1979, 1998), que apostaba por el valor de la cita como medidor de la calidad del trabajo científico, y la 'constructivista', que reparaba en el valor retórico que adquieren las citas dentro del texto como recurso argumentativo mediante el cual poder persuadir al lector (Gilbert, 1977; Latour, 1987).

Los primeros intentos por estudiar los motivos y las razones por las cuales se citan los textos de otros autores parten de los científicos de la información (Garfield, 1964; Hodges, 1972), quienes establecen listados sobre los motivos que encuentra el escritor de un texto académico para citar. Sin embargo, la primera tipología sistemática realizada a partir de un análisis de los contenidos de las citas, se la debemos a dos sociólogos de las ciencias, Moravcsik y Murugesan (1975). Otros muchos autores seguirán con posterioridad esta taxonomía en sus trabajos (Chubin \& Moitra, 1975; Oppenheim \& Renn, 1978; Cano, 1989), incluso desde la lingüística (Swales, 1986; Teufel, Siddharthan \& Tidhar, 2006).

Dentro de la Lingüística, las clasificaciones comenzaron a hacerse desde una perspectiva formalista, de modo que en los estudios (Hyland, 1999; Thompson, 2001; Mayor Serrano, 2006; Petrić, 2007; Okamura, 2008; Samraj, 2008) se partía de la distinción establecida en 1990 por Swales entre citas integrales (aparecen en el cuerpo del texto) y no integrales (aparecen entre paréntesis o en nota al pie). En estos estudios no se reparaba tanto en las razones que encuentra el escritor para citar una fuente como en la función retórica que cumple la cita dentro del texto. Thompson (2001) es el primero en desgajar la clasificación de Swales (1990) en diferentes funciones retóricas de citas que afectan de modo distinto al escrito y que tienden a clasificarse en las distintas secciones del texto académico en función de su retórica, es decir, habrá ocasiones en las cuales, debido al tono requerido por la sección, se emplearán funciones expositivas sobre el conocimiento referido de otros autores, mientras que en otras primará un uso de las fuentes persuasivo, argumentativo, etc. Thompson (2001) mostró en su investigación que el uso de las funciones de las citas hechos por doctorandos en sus tesis variaban en función de la disciplina estudiada, la Botánica Agrícola o la Economía Agroalimentaria. Diferentes estudios corroboran esta variabilidad interdisciplinaria, así como otros traen a colación la variabilidad intradisciplinaria en las prácticas de citación (Chubin \& Moitra, 1975; Moravcsik \& Murugesan, 1975; Hyland, 1999; Thompson, 2001; Petrić, 2007; Harwood, 2009).

Otro contexto en el que se distingue el uso de las funciones de las citas es el que se da entre los escritores profesionales y los no profesionales. Schembri (2009) sintetiza esta distinción en la necesidad que tiene el experto de citar para demostrar que sus resultados son nuevos, importantes y verdaderos, frente al escritor no experto, que tiende a utilizar la cita para exponer el conocimiento del campo que está tratando en su investigación. La impericia en el manejo de la citación con valor argumentativo o persuasivo (Latour, 1987; Laca, 2001) limita sus escritos hacia un 
discurso referido en el que la cita tiene principalmente una función atributiva (véase 3.1.), en la cual se reproducen fielmente las ideas sugeridas en la fuente consultada (Latour, 1987; Dong, 1996; Schembri, 2009). El autor profesional hará un esfuerzo por contrastar los documentos citados y establecerá relaciones entre ellos, de modo que exista una transformación de la información recibida, convirtiéndola de este modo en conocimiento nuevo (Dong, 1996; Petrić, 2007; Kiczkovsky, 2008), con lo que consigue imponer su voz con autoridad sobre la de las fuentes citadas que refiere, en ocasiones también mediante la emisión de juicios y valoraciones sobre el trabajo citado (Hyland, 2000; Mostacero, 2004; Thompson, 2005; Charles, 2006; Castelló, Corcelles, Iñesta, Bañales \& Vega, 2011).

\section{Corpus utilizado y metodología}

El presente trabajo se ha centrado en el análisis de las funciones de las citas dentro de la memoria de máster desde una perspectiva discursiva y retórica, dentro del campo de la Lingüística Aplicada. El análisis textual de las funciones retóricas de las citas aplicado al estudio retórico contrastivo de la escritura académica en la cultura española y filipina, ha sido el objetivo del análisis realizado en la presente investigación. Todos los informantes del grupo F son escritores filipinos del Departamento de Lenguas Europeas de la Universidad de Filipinas, bilingües en inglés y otra/s lengua/s nativa/s que se hablan en el archipiélago. Los integrantes del grupo E tienen como lengua materna el español y escriben su memoria en esta lengua.

El corpus analizado consta de 14 textos, 7 memorias escritas en español por estudiantes filipinos de ELE que conforman el grupo F, y otras 7 escritas por nativos españoles, el grupo E. La temática de las mismas versa sobre asuntos relacionados con la adquisición de segundas lenguas, dentro de la disciplina de la Lingüística Aplicada.

La etiqueta identificativa de cada informante se establece por nacionalidad ( $\mathrm{F}=$ filipino; $\mathrm{E}=$ español) y orden numérico de la memoria analizada $(1,2,3,4,5,6$, 7). Así, F1 se corresponde con el primer informante del grupo filipino y E7 con el séptimo del grupo español.

En el procedimiento de análisis seguido en esta investigación, primeramente se han identificado las pistas lingüísticas en el contexto de la cita para su clasificación. Para ello se han considerado tanto la significación de los verbos introductorios empleados como las marcas circundantes ('por ejemplo', 'p.e.', 'vid.', 'véase', etc.). Sin embargo, para otras categorías en las cuales la identificación lingüística se hace más compleja, como EN, AT o AP, se ha considerado el contenido de la cita para proceder a su etiquetado. Después se han codificado las citas y se han ordenado en tablas en las que se ha cuantificado cada categoría clasificada.

En el recuento de los datos que se ha llevado a cabo en el análisis se ha optado por trabajar con el criterio de la densidad de citas, es decir, la medida de la frecuencia de citas por cada 1.000 palabras, con el fin de ofrecer datos comparables entre las 
distintas memorias analizadas en igual proporción. Del mismo modo, este tipo de procedimiento, utilizado previamente en otras investigaciones sobre citación por Hyland (1999, 2000), Thompson (2001, 2005) o Petrić (2007), nos permite establecer comparaciones con estos autores.

\section{Tipología de las funciones retóricas de las citas}

Se ha comprobado empíricamente que no existe una tipología de citas estándar, sino que esta varía según la disciplina en la que se realice el análisis (White, 2004; Petrić, 2007). Igualmente otros estudios (Mayor Serrano, 2006; Petrić, 2007) advierten de la variabilidad de género dentro de la disciplina y de cómo esto afecta también a la selección de las citas. Por ello, en su artículo, Petrić (2007) comenta la dificultad de adaptar enteramente la tipología formalista creada por Thompson (2001) y en la que basa su taxonomía para clasificar las funciones de las citas en su estudio, por proceder del análisis de otra disciplina. Este autor partía de la distinción clásica de Swales (1990) sobre las citas integrales y no integrales, y subdividía las no integrales en cinco categorías: source, identification, origin, reference y example. Petrić (2007) las ampliaría a nueve, attribution, exemplification, further reference, statement of use, application, evaluation, establishing links between sources, comparition of one's own finding or interpretation with other sources $y$ others. En el caso particular del presente trabajo, se ha optado por crear una nueva tipología de análisis a partir de la clasificación de Petrić (2007). Sin embargo, nuestro estudio contempla ciertas particularidades (véase 3.1., 3.7. y 3.9.) con respecto a las funciones introducidas por esta autora.

En la tipología que se presenta a continuación se han incluido ejemplos-codificados entre paréntesis- de las memorias de máster analizadas en la investigación. Dentro de los paréntesis aparece la etiqueta que identifica al autor de la tesis, la nacionalidad (E o F) y el número adscrito $(1,2,3,4,5,6,7)$, además de la sección ${ }^{2}$ en la que se ha encontrado la cita.

\subsection{Atribución (AT)}

Esta función es meramente descriptiva y confiere a la cita un valor informativo mediante el cual se declara que la opinión, idea o proposición que se incluye en el texto está en consonancia con la de otro autor que la ha referido anteriormente. De este modo, el escritor que utiliza este tipo de cita busca el sustento de una autoridad para reforzar una opinión dada y dotarla así de una mayor credibilidad ante los ojos del lector. De la misma manera, este tipo de cita reconoce implícitamente una deuda con el trabajo de un autor precedente.

Esta función puede presentarse en forma de paráfrasis, resumen o cita directa de las palabras de la fuente mencionada, de la cual se sirve el escritor en la exposición de un tema que guarda relación con otras lecturas previas que tratan sobre el mismo asunto. Por lo tanto, lejos de valorar el texto citado, la función AT tiene un marcado carácter descriptivo, como en los siguientes ejemplos: 
Las "Propuestas de Alicante" hacen hincapié en la importancia de la especialización de los profesores en la didáctica de las L2 para la efectividad de las medidas que se propongan en esta área educativa (Hernández, Roca \& Villalba, 2007). (F7-M.T.).

Normalmente AT se emplea dentro de la escritura académica para referirse a los trabajos que se han consultado y que conforman el material teórico de apoyo para la realización de un trabajo de investigación, dejando así constancia de su lectura. Por lo tanto, esta función abunda en las secciones más expositivas de la memoria de máster o de la tesis doctoral. Es esperable, por ello, su abundancia en las secciones de la tesis que tienen como finalidad la contextualización del trabajo y la exposición de la información, como son el marco teórico o la introducción.

\subsection{Ejemplificación (EJ)}

Esta función aporta información que sirve para ilustrar una afirmación del autor en el trabajo de investigación. La cita actúa aquí como una evidencia específica, apoyando una afirmación mediante la ampliación de una información dada. De este modo, la referencia incide en profundizar y reforzar la argumentación del escritor con elementos tomados de otros trabajos que pueden aplicarse al presente:

Alcántara Antonio (2006: 887) explicó que ha habido cambios o sustituciones. La e en español se ha cambiado a la vocal a, por ejemplo: ahora dice detalya [detályah], en vez de detalye. (F2-M.T.).

En esta función la cita suele ir precedida de 'por ejemplo', lo que contribuye a su fácil identificación en el texto. Esta función retórica puede aparecer tanto en citas integrales como en no integrales. Dentro de las no integrales, es frecuente que se relegue esta información a la nota al pie, debido a que el autor considera que esta aclaración no es relevante para el decurso de la idea e interrumpe la exposición.

\subsection{Referencia (REF)}

Mediante esta función retórica de la cita se redirige al lector a un trabajo previo, facilitándole la referencia del texto, con la finalidad de que este pueda ampliar la información apuntada en el trabajo de investigación sobre ese asunto. Este tipo de cita resulta especialmente útil para describir el contexto del estudio del escritor sin la necesidad de incluir en el texto la información considerada subsidiaria por el autor. Por lo tanto, resulta interesante también esta función para conocer el criterio de relevancia que el escritor aplica en su memoria y, como consecuencia, el conocimiento que atesora sobre el campo de estudio sobre el que está escribiendo. En otras palabras, el citador puede demostrar un uso experto al gestionar la cantidad justa de información que necesita manejar en la elaboración de su memoria de máster, dejando los contenidos menos relevantes documentados mediante REF. De esta manera, el conocimiento 
que aporta esa cita queda disponible para el lector curioso, que puede acceder a la fuente siempre que quiera para ampliar su saber, al mismo tiempo que el investigador prueba estar bien documentado.

Esta cita suele aparecer entre paréntesis y viene precedida normalmente por abreviaturas del tipo 'vid.', 'v.', 'c.f.' o formas verbales tales como 'ver' o 'véase', ocasiones todas ellas en las que tiende a ser una cita no integral:

Dicho de otra manera, mientras la continua expansión económica de los países desarrollados provoca la profesionalización de su población, se va dejando un vacío en ciertas áreas de la vida cotidiana, tales como la construcción, la labor doméstica, el servicio sanitario, etc. (véanse Cheng, 2004; Salazar Parreñas, 2001a). (F3-M.T.).

En ocasiones se omiten estas marcas en el texto, quizás porque los escritores no son expertos, no están siempre familiarizados con las normas de la escritura académica, las desconocen o las ignoran u obvian por no considerarlas relevantes. Sin embargo, también se puede usar de forma consciente la cita integral, como en los ejemplos anteriores, en los que la referencia se hace explícita.

\subsection{Declaración de uso (DE)}

Se usa para declarar que el trabajo citado o una de sus partes se ha utilizado en la memoria de máster. En este caso, suele detallarse el propósito para el cual se utiliza dentro de esta.

En la mayoría de los casos esta función retórica se reserva para el apartado de INTR, en la que se habla de modo general de los trabajos en los que se ha basado el autor para llevar a cabo su análisis. También tiene un uso destacado en la sección MET, en la que se describen los instrumentos utilizados para realizar el análisis, o en CON o en los resúmenes de los capítulos, de forma retrospectiva. Pueden darse casos tanto de citación integral como de no integral.

La clasificación según la forma del input responde a la tipología propuesta por Dominique Macaire y Wolfram Hosch, recogida por Ch. Cuadrado, Y. Díaz y M. Martín (1999) (E4-MET).

No obstante, la delimitación de las fronteras entre DE y la función que se estudiará a continuación, AP, no está del todo clara y puede generar problemas en la decisión de clasificar las citas bajo una u otra categoría.

\subsection{Aplicación (AP)}

Este tipo de cita establece conexiones entre el citador y el trabajo citado con el objetivo de usar los argumentos, los conceptos, la terminología o los procedimientos 
desde el trabajo citado, para utilizarlos con un objetivo propio en la memoria de máster (Petrić, 2007).

En el siguiente ejemplo, la cita con función retórica AP establece uno de los objetivos del trabajo a través del concepto prestado de otra obra. Prima en ella el uso que de la cita hace el citador, F3, quien hereda una idea procedente de Kasper y BlumKulka y de Larsen-Freeman y Long y la adapta de forma explícita a su memoria, para aplicar esos conceptos al objetivo del escrito.

Puesto que los antecedentes circunstanciales de una persona inciden sobre la utilización de una determinada lengua (Kasper \& Blum-Kulka, 1993; LarsenFreeman \& Long, 1994), queremos perfilar a los inmigrantes filipinos en España en general y caracterizar la trayectoria profesional que han seguido nuestros informantes desde sus estudios hasta su actual empleo en el país (F3INTR).

La función de este tipo de cita está muy próxima a AT, por lo que resulta ciertamente complicado cuantificar estas citas dentro de la taxonomía que se está descubriendo en este apartado.

\subsection{Evaluación (EV)}

Con esta función retórica se evalúa el trabajo de otro autor a través de los comentarios del citador. Aunque en la mayoría de las citas las valoraciones tienden a reconocer los méritos de las fuentes de forma positiva, las críticas negativas también aparecen. Sin embargo, cuando estas se realizan, tal y como advertían Moravcsik y Murugesan (1975) y Brooks (1985) en sus trabajos, la cita negativa suele ir precedida de una evaluación positiva previa.

Junto a las innegables virtudes que atesora [S. Fernández], esta investigación oculta en mi opinión algunos defectos de naturaleza metodológica que merecen atención. En primer lugar, para la recogida de datos se contó con la división en cursos que hace para la Escuela Oficial de Idiomas de Madrid, y más concretamente con los cursos $2^{\circ}, 3^{\circ}$ y $4^{\circ}$, que, teóricamente, debían corresponder al nivel medio bajo, medio alto y avanzado, respectivamente (E6M.T.).

En esta cita, el informante no entra a valorar la opinión del autor, pero pone en duda su afirmación sin cuestionarla abiertamente. El desacuerdo de opinión es también una evaluación del trabajo citado, aunque no pueda considerarse como una crítica negativa de forma explícita.

Aunque para nosotros, la interferencia lingüística es la combinación de lo que dicen Escobar y Thomason y Kaufman (F2-M.T.). 
Como ya se comentó en REF (véase 3.3.), esta función retórica denota la adopción de un punto de vista crítico de una persona que conoce la materia que trata y que se atreve a emitir juicios al respecto, valorando sus fuentes con criterio y autoridad, en lugar de conformarse con hacer un uso enciclopédico expositivo de los textos referidos. En este sentido, la función EV guarda una estrecha relación con la argumentación, mientras que otras funciones retóricas, como la AT, COMP o REF están más cercanas a la exposición.

\subsection{Enlaces entre las fuentes (EN)}

La función de esta cita es señalar enlaces, comparaciones y contrastes entre las fuentes usadas. Este tipo de cita se utiliza para mostrar diferencias en los puntos de vista sobre un tema, así como para manifestar que el investigador es capaz de identificar asuntos controvertidos (Petrić, 2007).

Justo lo que Nelson (1992: 327) sostiene que "al acercarse a un idioma trasplantado a un nuevo contexto cultural y lingüística, se lleva una a las diversas realizaciones de la noción de la lengua y las variedades que pueden desarrollar un lenguaje". Esto puede ser demostrado, por ejemplo, por la existencia del taglish e inglés de Filipinas. Hay una gran afluencia de palabras ingleses se ha asimilado en el tagalo y otros idiomas nativos que resulta taglish (que se refiere a tagalo infundido con términos de inglés americano). Sin embargo, hay un debate sobre si existe diglosia o bilingüismo, o incluso semilingualismo (Martin-Jones, 1986), entre el filipino y el inglés (F2-M.T.).

Como se aprecia en esta cita, en EN pueden aparecer tanto citas integrales como no integrales. En el contraste que se establece entre las diferentes perspectivas que adoptan los autores para definir un término puede observarse también esa madurez escritora del autor, pues mediante esta suma de opiniones y sus comentarios y conclusiones hacia estas definiciones yuxtapuestas, está transformando el conocimiento que le llega por medio de las fuentes consultadas, creando conocimiento nuevo (Dong, 1996). Este proceso lleva implícito un componente importante de argumentación, ya que se hace una selección de los trabajos que interesan para la memoria -dejando otros al margen-, se contrastan y se sintetizan las posiciones encontradas para hallar la definición más apropiada a la especificidad gnoseológica de la memoria. Esto ocurre también con el siguiente ejemplo, en el que se aclara una cuestión con respecto al contenido fundamental en la configuración del tema que se está tratando:

Como se puede ver, Weinreich dejó abierta la cuenta psicológica de la identificación interlingual, por la cual Selinker junto con Lamendella, en 1978, postularon una estructura oculta del cerebro que se activaría cuando el aprendiz intentara expresar significados en la LM. Adicionalmente, propusieron que debido a dicha estructura oculta, hagan lo que hagan los aprendientes, aún 
fosilizarán algunas partes de su IL. Pienemann y sus colegas (En: Selinker, 1992) suponen que esa fosilización es inevitable porque cada aprendiz construye su propia gramática. Por la realidad de fosilización de estructuras desemejantes con la L1, Selinker así opina que la L1 no es enteramente el punto de partida de la adquisición de la LM (F1-M.T.).

En estos ejemplos se observa que EN no es una categoría que solo registra las polémicas en las que se oponen y enfrentan las ideas de los autores, sino que también está abierta a una actitud colaborativa en la cual se construye conocimiento nuevo a partir del contraste de las ideas de distintos escritores. En estos casos, el citador entreteje las referencias a los trabajos citados, relacionando las ideas de distintos autores entre sí, lo que aporta una fuerza mayor al argumento tratado y da más brillo al investigador que utiliza este recurso. De ello se infiere que maneja de forma adecuada la bibliografía y que puede atribuir ideas a un autor y contrastarlas de forma organizada con las de otros investigadores que han opinado a ese respecto, enriqueciendo el concepto tratado con la aportación de distintos matices. Sin embargo, esta no es una categoría que solo registra las polémicas en las que se oponen y enfrentan las ideas de los autores, sino que también está abierta a una actitud colaborativa en la cual se construye conocimiento nuevo a partir del contraste de las ideas de distintos escritores. En estos casos, el citador entreteje las referencias a los trabajos citados, relacionando las ideas de distintos autores entre sí, lo que aporta una fuerza mayor al argumento tratado y da más brillo al investigador que utiliza este recurso. De ello se infiere que maneja de forma adecuada la bibliografía y que puede atribuir ideas a un autor y contrastarlas de forma organizada con las de otros investigadores que han opinado a ese respecto, enriqueciendo el concepto tratado con la aportación de distintos matices.

\subsection{Comparación de los resultados propios o interpretaciones propias sobre un asunto, con otras fuentes (CO)}

Este tipo de cita se utiliza para indicar similitudes o diferencias entre los trabajos de un autor y de otro, típicamente cuando discuten los resultados encontrados en el análisis (Petrić, 2007). Esta función resulta de gran utilidad para adquirir una visión global de cómo se ha tratado el tema investigado en el campo, pues cada investigación supone una contribución al mismo y el contraste de los resultados de los estudios llevados a cabo sobre un mismo tema propicia una mirada más amplia sobre ese aspecto. En unos casos se confirmarán los datos obtenidos en la investigación. Por el contrario, en otros, podría producirse una contradicción entre los resultados de distintos trabajos, hecho que sugiere una revisión adecuada sobre la metodología empleada en los estudios y el incentivo de nuevas investigaciones sobre ese fenómeno investigado que confirmen los datos obtenidos.

Confirmamos con estas cifras la observación de Ortega Masagué (2005: 6 \& 9) citada en la revisión bibliográfica (F3-RES). 
Como se ha mencionado, las citas con esta función retórica suelen aparecer en las secciones de RES, en la Discusión y en CON, apartados en los que se contrastan los resultados. Estas citas pueden ser tanto integrales como no integrales, aunque su naturaleza sea típicamente integral, ya que, al igual que DE, sirven para resaltar la investigación del autor y su posición respecto a los otros trabajos citados.

\subsection{Competencia (COMP)}

La función retórica COMP consiste en concentrar una lista de citas de varios autores en torno a una afirmación. Se utiliza para resaltar la relevancia de un concepto, término, idea o afirmación dentro de la investigación, es decir, la información que es considerada como conocimiento general en el campo que se está investigando. Del mismo modo, sirve para demostrar el dominio del investigador con respecto a un tema, pues prueba con el listado de citas haber leído la bibliografía disponible sobre ese tema.

Las muestras de lengua meta a las que está expuesto el alumno desempeñan un papel muy importante en el proceso de aprendizaje-adquisición de una lengua extranjera, tal y como demuestran los estudios realizados por Krashen (1985) y McLaughlin (1987) y continuados por Bialystock (1990) (E4-M.T.).

Esta función es similar a AT, con la única diferencia del énfasis y la intensidad puestas en la afirmación con el refuerzo en el número de las citas, que sirve para afianzar de forma sólida el concepto descrito con la autoridad de una serie de autores que han tratado previamente el tema. Por su parte, Petrić (2007) incluye este uso dentro de EN. Para nosotros, COMP cumple una función distintiva que no se asemeja a la de EN, pues en ella no existe el contraste, la oposición o la acumulación de información, sino que trata de reforzar el conocimiento disciplinar en torno a una afirmación.

\section{Resultados}

\subsection{Resultados globales sobre el número y la frecuencia del total de las citas}

En el corpus analizado se han realizado un total de 2.100 citas, repartidas entre los dos grupos descritos, 1.248 citas por parte del filipino y 852 por el español. La media de citas por tesis en el caso del grupo filipino es de 178 citas por memoria, mientras que en el español es ligeramente inferior, 122 citas por obra. Este dato ha de ser contrastado con el número de palabras, que en el grupo filipino alcanza las 241.589 palabras, con 34.513 palabras promedia por memoria, más elevada que en el español, que cuenta con 209.599 palabras por cada trabajo, con una media de 29.942 palabras por memoria. El contraste entre el número de citas y el número de palabras empleado en la redacción de estas obras indica que los filipinos utilizan como promedio una 
cita por cada 245 palabras, mientras que en el grupo español la cita aparece cada 323 palabras, en un índice de frecuencia menor que en el grupo filipino. Este dato se corrobora con el cálculo de la densidad de las citas por cada 1.000 palabras, que es más alto en el caso del grupo filipino de 5,2, con respecto al español de 4,1.

En la Tabla 1 que se muestra a continuación, se ofrece un resumen cuantitativo de la densidad de las funciones de las citas por informante, cuya suma aporta datos también sobre la densidad de los grupos y los totales de las funciones de citas recogidos en el corpus estudiado:

Tabla 1. Densidad de citas por grupo, informante y función retórica de la cita.

\begin{tabular}{|l|c|c|c|c|c|c|c|c|c|c|c|}
\hline & AT & AP & DE & COMP & CO & REF & EJ & EV & EN & \%parcial & \%total \\
\hline F1 & 2,3 & 0,6 & 0,3 & 0 & 0 & 0 & 0,04 & 0,08 & 1,6 & 12,4 & 7,1 \\
\hline F2 & 2,5 & 0,2 & 0,1 & 0,4 & 0,6 & 0,02 & 0,09 & 0,04 & 0,9 & 12,1 & 6,8 \\
\hline F3 & 1,9 & 1,2 & 0,03 & 2,9 & 0,3 & 1,1 & 0,2 & 0,1 & 3,5 & 28,2 & 16,2 \\
\hline F4 & 1,7 & 0,3 & 0,1 & 0 & 0,05 & 0,6 & 0,05 & 0 & 0,7 & 8,9 & 5,1 \\
\hline F5 & 1,6 & 0,05 & 0 & 0 & 0 & 0,05 & 0 & 0 & 0 & 4,3 & 2,4 \\
\hline F6 & 1,4 & 2,1 & 0,8 & 0,9 & 0,1 & 0,05 & 0,9 & 0 & 3,2 & 23,9 & 13,7 \\
\hline F7 & 2,6 & 0,3 & 0,2 & 0 & 0 & 0,03 & 0,04 & 0 & 0,9 & 10,2 & 5,8 \\
\hline Ftotal & $\mathbf{2 , 2}$ & $\mathbf{0 , 5}$ & $\mathbf{0 , 2}$ & $\mathbf{0 , 5}$ & $\mathbf{0 , 2}$ & $\mathbf{0 , 2}$ & $\mathbf{0 , 1}$ & $\mathbf{0 , 0 3}$ & $\mathbf{1 , 3}$ & 100 & 100 \\
\hline \% & $\mathbf{4 2 , 2}$ & $\mathbf{9 , 5}$ & $\mathbf{3 , 8}$ & $\mathbf{9 , 5}$ & $\mathbf{3 , 8}$ & $\mathbf{3 , 8}$ & $\mathbf{1 , 9}$ & $\mathbf{0 , 5}$ & $\mathbf{2 5}$ & - & - \\
\hline E1 & $\mathbf{6 , 5}$ & 0,2 & 0,07 & 0,5 & 0 & 0,07 & 0,07 & 0,04 & 0,9 & 28,4 & 12,2 \\
\hline E2 & $\mathbf{1 , 1}$ & 0,2 & 0,4 & 0 & 0 & 0,1 & 0,1 & 0,1 & 0,4 & 7,1 & 3,1 \\
\hline E3 & 2,9 & 0,4 & 0,2 & 0 & 0,03 & 0 & 0,03 & 0,2 & 0,5 & 14,2 & 6,1 \\
\hline E4 & 1,6 & 0,03 & 0,08 & 0,3 & 0 & 0 & 0,08 & 0 & 0,6 & 9,1 & 3,8 \\
\hline E5 & 0,7 & 1,5 & 0,5 & 1,5 & 0,5 & 0,09 & 0,4 & 0,05 & 2,1 & 25,7 & 11,1 \\
\hline E6 & 0,4 & 0,5 & 0,03 & 0 & 0,09 & 0,5 & 0,03 & 0,2 & 0 & 5,7 & 2,4 \\
\hline E7 & 1,7 & 0,3 & 0 & 0,09 & 0 & 0 & 0,03 & 0,03 & 0,7 & 9,8 & 4,2 \\
\hline Etotal & $\mathbf{2 , 2}$ & $\mathbf{0 , 4}$ & $\mathbf{0 , 1}$ & $\mathbf{0 , 3}$ & $\mathbf{0 , 0 9}$ & $\mathbf{0 , 1}$ & $\mathbf{0 , 0 9}$ & $\mathbf{0 , 0 9}$ & $\mathbf{0 , 7}$ & 100 & 100 \\
\hline \% & $\mathbf{5 3 , 7}$ & $\mathbf{9 , 8}$ & $\mathbf{2 , 5}$ & $\mathbf{7 , 4}$ & $\mathbf{2 , 3}$ & $\mathbf{2 , 5}$ & $\mathbf{2 , 3}$ & $\mathbf{2 , 3}$ & $\mathbf{1 7 , 2}$ & - & - \\
\hline TOTAL & $\mathbf{2 , 2}$ & $\mathbf{0 , 4}$ & $\mathbf{0 , 2}$ & $\mathbf{0 , 1}$ & $\mathbf{0 , 0 4}$ & $\mathbf{0 , 2}$ & $\mathbf{0 , 1}$ & $\mathbf{0 , 0 5}$ & $\mathbf{1 , 1}$ & - & - \\
\hline \% & $\mathbf{5 0}$ & $\mathbf{9 , 1}$ & $\mathbf{4 , 6}$ & $\mathbf{2 , 3}$ & $\mathbf{0 , 9}$ & $\mathbf{4 , 6}$ & $\mathbf{2 , 3}$ & $\mathbf{1 , 2}$ & $\mathbf{2 5}$ & - & - \\
\hline
\end{tabular}

El cálculo numérico de citas en AT marca una diferencia considerable con el resto de funciones retóricas utilizadas en las citas. El 50\% del total se establecen en la categoría AT y entre AT $(50 \%)$ y EN $(25 \%)$ se reparten las tres cuartas partes de las citas recogidas en el corpus. Estas dos son las funciones retóricas predominantes utilizadas en las memorias de máster, siendo el resto muy inferiores en cantidad y en representación. 


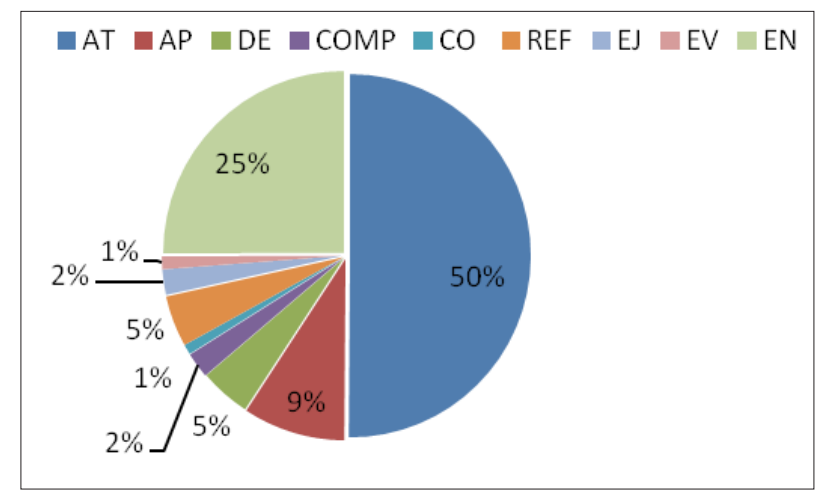

Gráfico 1. Porcentaje sobre la densidad de citas por la función retórica de la cita.

En el siguiente apartado se examinará el reparto de estas funciones entre ambos grupos y entre cada uno de los informantes, en busca de su contraste y diferencia.

\subsection{Resultados de la comparación entre los grupos sobre la frecuencia en las funciones retóricas de las citas por informante}

Al comparar los datos referidos a las funciones de las citas utilizadas por los informantes filipinos y españoles, se advierte que existe una equivalencia generalizada en todas las categorías analizadas. De todas ellas, existe una mínima superioridad en el uso de las funciones en el grupo F sobre el E, excepto en la categoría de AT, en el que se igualan los resultados y en $\mathrm{EV}$, en las que se observan tendencias inversas:

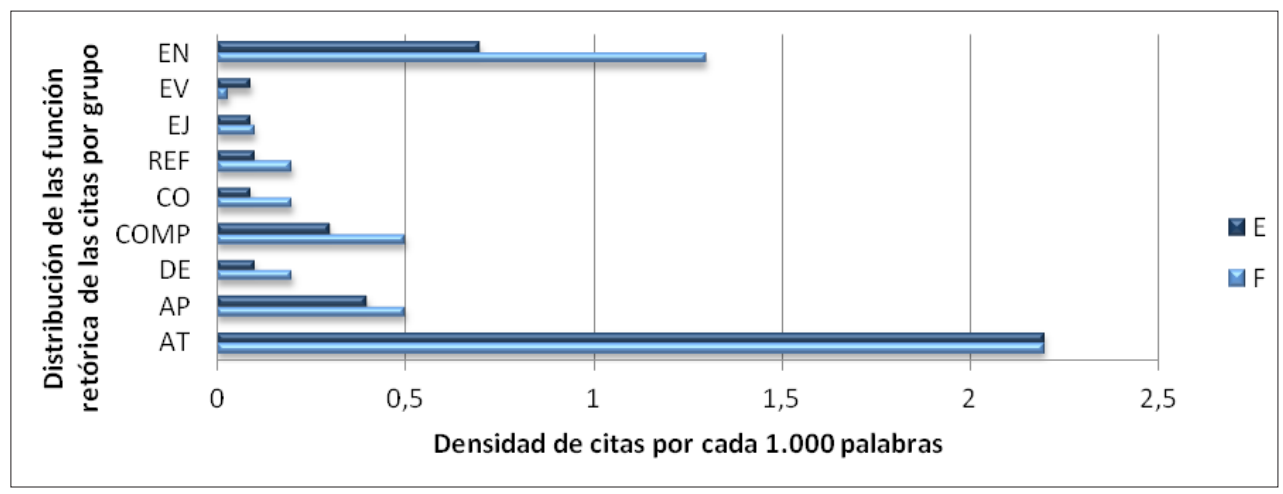

Gráfico 2. Densidad de la función retórica de la cita por grupos.

En el caso de EN, hay una pronunciada superioridad en los datos por parte del grupo F sobre el E, 1,3> 0,7. Como se refiere en la tipología (3.7.), EN es una categoría en la que el investigador muestra la transformación del conocimiento adquirido, y por lo tanto el dominio en el factor académico del uso de la información referida de las fuentes. En EV aparece el único caso en el que la tendencia general se invierte, 
es decir, el grupo E $(0,09)$ supera en densidad de citas al grupo $\mathrm{F}(0,03)$, que en datos reales se manifiesta en un $17>7$ en números de citas.

\subsection{Resultados en la comparación entre los informantes en la frecuencia de las funciones retórica de las citas por informante}

En el Gráfico 3 correspondiente a los resultados individuales no se aprecian diferencias significativas en las que coinciden todos los miembros de un grupo y que los distingan del otro.

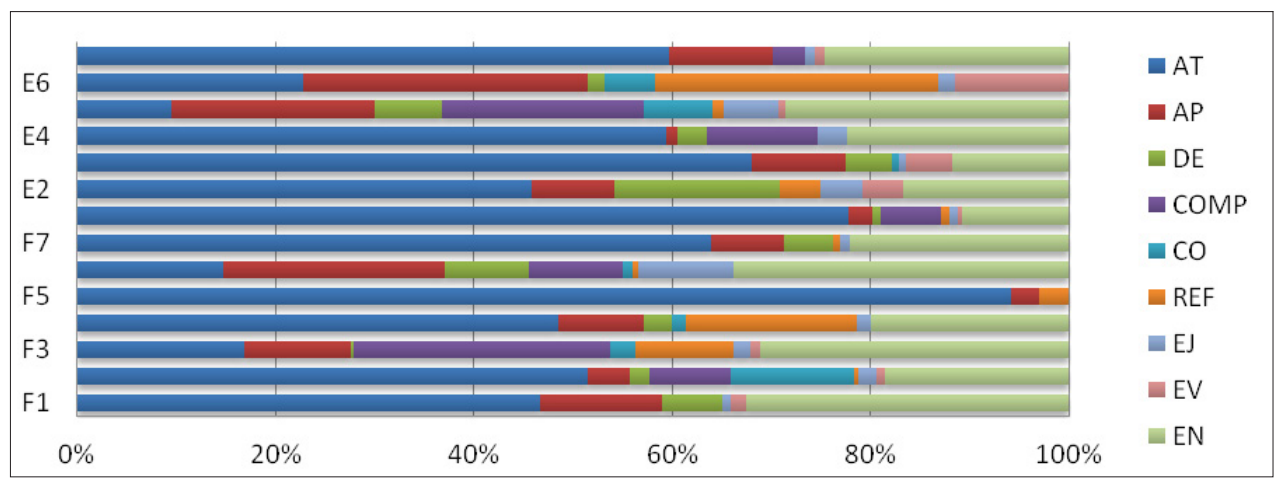

Gráfico 3. Porcentaje de la función retórica de las citas por informantes.

Se observan más bien diferencias particulares entre los individuos, pero no así entre las nacionalidades estudiadas. Solo se aprecia un uso generalizado mayor de EV en el caso del grupo E (E1: 0,04; E2: 0,1; E3: 0,2; E5: 0,05; E6: 0,2; E7: 0,3) sobre F (F1: 0,08; F2: 0,04; F3: 0,1). En F solo la suma de F1 (36,4\%) y F3 (45,4\%) supone más del 80\% del total de citas EV dentro del grupo. Del mismo modo, CO tiene una mayor presencia en el grupo F (F2: 0,6; F3: 0,3; F4: 0,05; F6: 0,1) que en el español (E3: 0,03; E5: 0,5; E6: 0,09). A pesar de estas diferencias poco significativas, no hay una función en la que domine absolutamente un grupo sobre el otro.

En la gran mayoría de las memorias la función predominante es AT (F1, F4, F5, F7, E1, E2, E3, E4, E7). Además, en algunas de ellas esta función se complementa con un alto índice en COMP (F3, F6, E1, E5) y EN (F1, F2, F3, F4, F6, F7, E1, E2, E3, E4, E5, E7), que al igual que AT se utiliza en la exposición de contenidos y en el reconocimiento al trabajo de la obra de otro autor. De hecho, los trabajos de F3, F6 y E5 contienen una densidad de citas mayor en la función EN que en AT. Un caso curioso y aislado es el de E6, en cuya investigación dominan las funciones AP $(0,5)$ y $\operatorname{REF}(0,5)$ sobre AT $(0,4), \operatorname{COMP}(0)$ y EN (0). El mayor uso de unas funciones sobre otras está relacionado con el propio carácter de la investigación. Es decir, hay algunos estudios en los que el autor prima el contenido teórico sobre el análisis, porque la finalidad de su trabajo es la de establecer una propuesta didáctica 
a partir de las teorías descritas por los otros autores citados en las fuentes. En ellas destacan cuantitativamente las funciones AT, COMP y EN. A pesar de que no existen diferencias evidentes y significativas entre los grupos, si es posible destacar diferencias intragrupales producidas por el uso particular de las citas que hacen algunos individuos en concreto, como en el caso claro de F3. En el Gráfico 3 se advierte que es también el que utiliza un mayor número de funciones retóricas en las citas repartidas equitativamente entre las distintas secciones de la memoria (AT: 1,9; AP: 1,2; DE: 0,03; COMP: 2,9; CO: 0,3; REF: 1,1; EJ: 0,2; EV: 0,1; EN: 3,5). En oposición a este informante, $\mathrm{F} 5$ presenta un valor bajo tanto de número de citas como de frecuencia en su texto, así como un peor reparto entre las funciones. Predomina el uso que hace de AT $(1,6)$ en su memoria, y solo incluye otras dos categorías, $\operatorname{AP}(0,05)$ y $\operatorname{REF}(0,05)$ en un porcentaje muy inferior.

En E1 y E4 también se identifica un abuso del AT y poca variedad en la función retórica de las citas usadas en la investigación. Por el contrario, el informante E6, que tenía un bajo índice de citas tanto en número como en frecuencia, utiliza una mayor variedad en las funciones y de un modo más proporcionado (AT: 0,4; AP; 0,5; DE: 0,03; CO: 0,09; REF: 0,5; EJ: 0,03; EV: 0,2). Sin embargo, el uso de las funciones de citas más variado y proporcionado en el grupo E es el de E5 (AT: 0,7; AP: 1,5; DE: 0,5; COMP: 1,5; CO: 0,5; REF: 0,09; EJ: 0,4; EV: 0,05; EN: 2,1).

Se observa en los datos recogidos en la tabla 1 la significación que tienen estos usos, y como el predominio de unas funciones sobre otras dentro de una memoria puede definir las características de esta, ya que de acuerdo con los objetivos que se persigan en la investigación, esta puede ser más teórica, más enfocada al análisis (DE y AP) o más centrada en contrastar los datos con otro estudio (CO).

\section{Discusión}

Los resultados expuestos anteriormente no han de ser tomados como hechos absolutos, sino como tendencias, pues no se puede hablar de conclusiones definitivas a partir de dichos resultados por el hecho de que se ha trabajado en el análisis con un corpus muy reducido. En síntesis, se ha mostrado en el análisis de este trabajo que existe un diferente comportamiento retórico por parte de los dos grupos analizados. Es el grupo F, el que ha obtenido un mayor número y densidad de citas en general, aunque los resultados varían entre los informantes de los dos grupos. En este sentido, algunos autores han hablado de que existen diferencias interdisciplinarias en los estudios académicos de citas, y lo han evidenciado con sus investigaciones, como Hyland (1999, 2000), Okamura (2008) o Harwood (2009). Las de Hyland (1999, 2000) y Harwood (2009), además de dar cuenta de este fenómeno, han referido también las diferencias intradisciplinarias que se registran en los estudios. A lo largo del análisis del presente estudio se ha evidenciado esta intradisciplinaridad, que se manifiesta en los distintos resultados que obtienen los informantes en esta investigación en 
cuanto al uso de las citas y al número y densidad de las funciones retóricas de las citas empleadas en cada sección, que apuntan a una mayor (F3, F6, E5) o menor (E1, E4, F5) calidad en el manejo de las citas, distinguiendo así a unos estudiantes de otros por la cantidad y la variedad de citas que incorporan a su escrito académico. Sin embargo, apenas se han observado diferencias transculturales entre los grupos, más allá del predomino del grupo F en la emisión de citas, solo compensado en AT y superado por el grupo E en EV.

Sobre las funciones retóricas de las citas, se ha hallado el dato en el estudio de que solo dos de ellas, AT y EN, aglutinan el 75\% del resultado total. Esto reafirma la proposición de que, como comenta Petrić (2007), el género de memoria de máster es primordialmente expositivo, en el que el autor trata de mostrar a la audiencia que está familiarizado con los contenidos del tema y de la disciplina que trata en su investigación. Entre los grupos analizados, solo se encuentra una diferencia relevante en la función EN, en la que el grupo F casi duplica al grupo E. Esto supone que el grupo F, además de atribuir a las fuentes el conocimiento que comenta en los textos, también contrasta la información obtenida entre los diferentes autores para transformar en conocimiento nuevo los contenidos teóricos referidos en la investigación (Dong, 1996; Petrić, 2007; Kiczkovsky, 2008).

Solo en un caso la densidad de citas es más alta, mínimamente, entre los escritores españoles que entre los filipinos. Esto sucede con la función EV, que aparece en seis de los siete informantes españoles, indicando una tendencia más propensa a la evaluación de los autores y de los contenidos referidos, en una actitud más argumentativa que la que presenta el grupo $\mathrm{F}$, cuyos trabajos se caracterizan por una alta exposición y descripción de las fuentes comentadas, pero una 'introspección’ pronunciada de la voz del autor de la investigación.

\section{CONCLUSIONES}

De los resultados comentados arriba se pueden extraer dos conclusiones generales en la investigación. La primera es que en español las memorias presentan rasgos de un estilo más argumentativo que las filipinas, que tienden a ser más expositivas. El informante español trata en su memoria de máster de ser más persuasivo e influir sobre la audiencia a la que está dirigida su investigación, intenta convencer sobre su verdad y expresar su opinión sobre las autoridades que consulta en relación al tema de su estudio, mientras que el filipino normalmente atribuye el conocimiento a los autores en los que se apoya de forma descriptiva. De igual modo, seis de los siete informantes del grupo E utilizan la función retórica de la cita EV, mayoritariamente en la sección de las conclusiones, con el fin de valorar, comentar o evaluar los trabajos de los autores citados. Por su parte, el grupo F hace un menor uso de EV, por lo que no se realiza una valoración de las obras consultadas e incluidas en su estudio, o se hace de forma indirecta, mediante el contraste de los autores con EN, pero generalmente 
no se muestra la opinión abiertamente en el texto de la investigación. En general, estos escritores renuncian a polemizar con las fuentes, a generar controversia o a hacer críticas negativas, aunque se dan algunos casos entre los informantes que manifiestan diferencias intragrupales en los que si las utilizan (F1, F2, F3). No obstante, ninguno de ellos hace uso de la cita negativa, mientras que en el grupo E la función EV se emplea en ocasiones para atacar un trabajo con el que no se está de acuerdo o que se quiere desprestigiar. Esto se debe a una diferencia cultural entre ambos grupos que se refleja en el uso de las citas, como comentan algunos autores sobre las normas de cortesía que rigen en la sociedad filipina, y que impiden manifestar abiertamente en público sus opiniones negativas sobre otros autores, por el prurito de mantener un ambiente armónico y sin confrontación con el resto de miembros de la comunidad disciplinaria a la que pertenecen (Maggay, 2002; Sales, 2007). Sin embargo, el grupo E manifiesta sus opiniones y valoraciones sobre las obras citadas sin ningún tipo de cortapisas culturales, por lo que los informantes se muestran mucho más directos en sus comentarios al valorar las referencias que aparecen en sus investigaciones.

La segunda conclusión general de la investigación tiene que ver con la relación que guardan las citas con la calidad en la memoria de máster. Se ha advertido que una mayor densidad de citas repartidas en distintas funciones retóricas es un indicador fiable que permite identificar a los buenos escritores. No es conveniente, sin embargo, confundir la cantidad elevada de citas en una investigación con la calidad del estudio. La cantidad ha de estar siempre justificada por un uso razonado en cada momento, en cada sección del trabajo del investigador. En el presente estudio, por ejemplo, se han identificado memorias que presentan una elevada frecuencia de citas de una función determinada. Este hecho está justificado, en ocasiones, por el tema en el que se centra el estudio, pues el enfoque teórico de una investigación, por ejemplo, va a exigir del escritor un número mayor de citas expositivas del tipo AT, COMP y EN, que un trabajo centrado en el análisis de variables con el fin de validar una hipótesis, en el que supuestamente habrá un número mayor de citas del tipo DE, AP o CO repartido por las distintas secciones. Esto es, los trabajos académicos no son buenos ni malos por la cantidad de citas que contienen, sino que la calidad de un trabajo viene determinada, en gran medida, por el adecuado empleo que se hace de las funciones retóricas de las citas en cada contexto retórico.

Por lo tanto, una investigación académica escrita por un universitario en una L2, o también en una L1, puede fracasar debido a un uso inadecuado de este elemento, lo que avala la necesidad de una didáctica de este recurso de la escritura académica. De este hecho deviene la conclusión de que una efectiva formación en este terreno permitirá al escritor hacer un uso más apropiado de las citas en el texto académico. A este respecto, Schembri (2009) comentaba en su estudio sobre las prácticas de citación como el grupo de estudiantes universitarios que recibía una instrucción formal específica en el uso de las citas en el discurso escrito obtenía mejores resultados. 
Es por ello que el estudiante extranjero que accede a una universidad para cursar estudios de grado o de posgrado, si espera tener éxito en esta empresa, estará obligado para ello a conocer las convenciones de la escritura académica y a respetar las reglas del juego que impone cada universidad, con el objetivo de superar las carencias que le puedan plantear las diferencias retórico-culturales de las que parte, es decir, las que ha adquirido durante su educación en una L1. 


\section{REFERENCIAS BIBLIOGRÁFICAS}

Bazerman, C. (2003). Intertextuality: How texts rely on other texts. En C. Bazerman \& P. Prior (Eds.), What writing does and how it does it: An introduction to analyzing texts and textual practices (pp. 83-96). Nueva Jersey: Erlbaum.

Brooks, T. A. (1985). Private acts and public objects: An investigation of citer motivations. Journal of the American Society for Information Science, 36, 223-229.

Cano, V. (1989). Citation behavior: Classification, utility, and location. Journal of the American Society for Information Science, 40, 284-90.

Castelló, M., Corcelles, M., Iñesta, A., Bañales, G. \& Vega, N. (2011). La voz del autor en la escritura académica: Una propuesta para su análisis. Revista Signos. Estudios de Lingüistica, 44(76), 105-117.

Charles, M. (2006). Phraseological patterns in reporting clauses used in citation: A corpus-based study of theses in two disciplines. English for Academic Purposes, 25(3), 310-331.

Chubin, D. E. \& Moitra, S. D. (1975). Content analysis of references: Adjunct or alternative to citation counting. Social Studies of Science, 5, 423-41.

Connor, U. (1996). Contrastive rhetoric. Cross-cultural aspects of second language writing. Cambridge: Cambridge University Press.

Cronin, B. (1998). Metatheorizing citation. Scientometrics, 43, 45-55.

Cumming, A. (1998). Theoretical perspectives on writing. Annual Review of Applied Linguistics, 18, 61-78.

Dong, Y. R. (1996). Learning how to use citations for knowledge transformation: Non-native doctoral student's dissertation writing in science. Researh in the Teaching of English, 30(4), 428-457.

Dudley-Evans, T. (2000). Genre analysis: A key to a theory of ESP? Ibérica, 2, 3-11.

Garfield, E. (1955). Citation indexes for Science. Science, 122(3159), 108-111.

Garfield, E. (1964). Science citation index': A new dimension in indexing. Science, 144(3619), 649-654.

Garfield, E. (1979). Is citation analysis a legitimate evaluation tool? Scientometrics, 1(4), 359-375.

Garfield, E. (1998). Random thoughts on citationology its theory and practice. Scientometrics, 43(1), 69-76. 
Gilbert, N. G. (1977). Referencing as persuasion. Social Studies of Science, 7, 113-122.

Harwood, N. (2009). An interview-based study of the functions of citations in academic writing across two disciplines. Journal of Pragmatics, 41(3), 497-518.

Hodges, T. L. (1972). Citation indexing: Its potential for bibliographical control. Tesis doctoral, University of California at Berkeley, California, Estados Unidos.

Hyland, K. (1999). Academic attribution: Citation and the construction of disciplinary knowledge. Applied Linguistics, 20(3), 341-367.

Hyland, K. (2000). Disciplinary discourses: Social interactions in academic writing. Harlow, Reino Unido: Longman.

Kiczkovsky, S. (2008). La monografía: Una perspectiva conceptual. En E. Arnoux (Ed.), Escritura y producción de conocimiento en las carreras de posgrado (pp. 103-120). Buenos Aires: Santiago Arcos.

Laca, B. (2001). Otras instancias enunciativas. En G. Vázquez (Coord.), Guía didáctica del discurso académico escrito: ¿Cómo se escribe una monografía? (pp. 81-94). Madrid: Edinumen.

Latour, B. (1987). Science in action. Cambridge, MA: Harvard University Press.

Maggay, M. P. (2002). Pahiwatig: Kagawiang Pangkomunikasyon ng Filipino. Quezon City: Ateneo de Manila University Press.

Mansourizadeh, K. \& Ahmad, U. K. (2011). Citation practices among non-native expert and novice scientific writers. Journal of English for Academic Purposes, 10, 152-161.

Mayor Serrano, M. B. (2006). La citación en la comunicación médica escrita (inglés español): Funciones y tipos. Lebende Sprachen, 51(2), 72-78.

Merton, R. K. (1942). The normative structure of science. En R. K. Merton (Ed.), The sociology of science: Theoretical and empirical investigations (pp. 267-278). Chicago: University of Chicago Press.

Moravcsik, M. J. \& Murugesan, P. (1975). Some results on the function and quality of citations. Social Studies of Science, 5, 86-92.

Mostacero, R. (2004). La construcción de la escritura personal a partir del discurso del otro. Lingua Americana, 15, 63-79.

Nicolaisen, J. (2007). Citation analysis. Annual Review of Information Science and Technology, 41(1), 609-641.

Okamura, A. (2008). Citation forms in scientific texts: Similarities and differences in L1 and L2 professional writing. Nordic Journal of English Studies, 7(3), 61-81. 
Oppenheim, C. \& Renn, S. P. (1978). Highly cited old papers and reasons why they continue to be cited. Journal of the American Society for Information Science, 29, 22531.

Parodi, G. (2007). El discurso especializado escrito en el ámbito universitario y profesional. Revista Signos. Estudios de Lingüistica, 40(63), 147-178.

Petrić, B. (2007). Rhetorical functions of citations in high and low-rated master's thesis. Journal of English for Academic Purposes, 6, 238-253.

Sales, M. J. (2007). La instrumentalidad de la cortesía verbal en la negociación de la identidad trasnacional de los emigrantes filipinos en España: Problemas interlingüisticos y propuestas didácticas. Tesis de magíster, Universidad de Valladolid, Valladolid, España.

Samraj, B. (2008). A discourse analysis of master's theses across disciplines with a focus on introductions. Journal of English for Academic Purposes, 7, 55-67.

Schembri, N. (2009). Citation practices: Insights from interviews with six undergraduate students at the University of Malta. Language Studies Working Papers, 1, 16-24.

Swales, J. (1986). Citation analysis and discourse analysis. Applied Linguistics, 7(1), 3956.

Swales, J. (1990). Genre analysis: English in academic and research settings. Cambridge, Inglaterra y Nueva York: Cambridge University Press.

Teufel, S., Siddharthan, A. \& Tidhar, D. (2006). Automatic classification of citation function. Ponencia presentada en the Conference on Empirical Methods in Natural Language Processing, Sydney, Australia.

Thompson, P. (2001). A pedagogically-motivated corpus-based examination of PhD theses: Macroestructure, citation practices and uses of modal verbs. Tesis doctoral, University of Reading, Reading, Reino Unido.

Thompson, P. (2005). Points of focus and position intertextual reference in $\mathrm{PhD}$ theses. Journal of English for Academic Purposes, 4, 307-323.

Venegas, R. (2006). La similitud léxico-semántica en artículos de investigación científica en español: Una aproximación desde el Análisis Semántico Latente. Revista Signos. Estudios de Lingüística, 39(60), 75-106.

Wette, R. (2010). Evaluating student learning in a university-level EAP unit on writing using sources. Journal of Second Language Writing, 19, 158-177.

White, H. D. (2004). Citation analysis and discourse analysis revisited. Applied Linguistics, 25(1), 89-116. 


\section{NOTAS}

1. Para una revisión general sobre este tema, pueden consultarse White (2004) y Nicolaisen (2007).

2. A lo largo de este trabajo se utilizarán las siguientes abreviaturas para referirse a las secciones de la memoria de máster: Introducción (INTR), Marco Teórico (MT), Método (MET), Resultados, (RES), Discusión (DIS), Propuesta Didáctica (P.D.) y Conclusiones (CON). 